Original Articles

\title{
Broca's region: A causal role in implicit processing of grammars with crossed non-adjacent dependencies
}

\author{
Julia Uddén ${ }^{\mathrm{a}, \mathrm{c}, *}$, Martin Ingvar $^{\mathrm{b}}$, Peter Hagoort ${ }^{\mathrm{a}, \mathrm{c}}$, Karl Magnus Petersson ${ }^{\mathrm{a}, \mathrm{c}, \mathrm{d}}$ \\ a Max Planck Institute for Psycholinguistics, Nijmegen, The Netherlands \\ ${ }^{\mathrm{b}}$ Stockholm Brain Institute, Department of Clinical Neuroscience, Karolinska Institutet, Stockholm, Sweden \\ ${ }^{\mathrm{c}}$ Donders Institute for Brain, Cognition and Behaviour, Centre for Cognitive Neuroimaging, Radboud University Nijmegen, The Netherlands \\ ${ }^{\mathrm{d}}$ Cognitive Neuroscience Research Group, Centre for Biomedical Research, Universidade do Algarve, Faro, Portugal
}

\section{A R T I C L E I N F O}

\section{Article history:}

Received 25 August 2015

Revised 14 February 2017

Accepted 14 March 2017

\section{Keywords:}

Artificial grammar learning

Non-adjacent crossed dependencies

Implicit learning

Broca's region

Transcranial magnetic stimulation

\begin{abstract}
A B S T R A C T
Non-adjacent dependencies are challenging for the language learning machinery and are acquired later than adjacent dependencies. In this transcranial magnetic stimulation (TMS) study, we show that participants successfully discriminated between grammatical and non-grammatical sequences after having implicitly acquired an artificial language with crossed non-adjacent dependencies. Subsequent to transcranial magnetic stimulation of Broca's region, discrimination was impaired compared to when a language-irrelevant control region (vertex) was stimulated. These results support the view that Broca's region is engaged in structured sequence processing and extend previous functional neuroimaging results on artificial grammar learning (AGL) in two directions: first, the results establish that Broca's region is a causal component in the processing of non-adjacent dependencies, and second, they show that implicit processing of non-adjacent dependencies engages Broca's region. Since patients with lesions in Broca's region do not always show grammatical processing difficulties, the result that Broca's region is causally linked to processing of non-adjacent dependencies is a step towards clarification of the exact nature of syntactic deficits caused by lesions or perturbation to Broca's region. Our findings are consistent with previous results and support a role for Broca's region in general structured sequence processing, rather than a specific role for the processing of hierarchically organized sentence structure.
\end{abstract}

(C) 2017 Published by Elsevier B.V.

\section{Introduction}

An important aspect of natural language processing is related to structured sequence processing (Christiansen \& Chater, 2008; Gomez \& Gerken, 2000; Petersson, Forkstam, \& Ingvar, 2004; Reber, 1967). The connection between sequence- and natural language processing is most easily described within the domain of syntax. During fluent syntax processing, structured sequences of words are parsed incrementally and, when possible, immediately. This process gains robustness by prediction of syntactic features of expected words downstream in a sentence. One example is number agreement in English. Plural marking of a noun (the boys rather than the boy) predicts that the corresponding verb will be in the plural form (play rather than plays). Sometimes words which are syntactically dependent, are far apart in terms of sequential order, that is, the dependency is non-adjacent or long-distance. For example, the noun-verb pair boys-play forms a non-adjacent dependency in

* Corresponding author at: Max Planck Institute for Psycholinguistics, PO Box 310, 6500 AH Nijmegen, The Netherlands.

E-mail address: Julia.udden@mpi.nl (J. Uddén).
"The boys in the morning group play with Susan". Multiple nonadjacent dependencies can also be found in a single sentence. For instance, in "All the animals that I can think of are wild", the dependent noun-verb pair "I-think" is embedded in the intervening material between the noun and verb in the pair "animals-are". The challenge of maintaining predictions based on multiple nonadjacent dependencies makes these structures well-suited for studying the neural implementation of structured sequence processing.

Recent research has investigated different types of sentence-level dependencies and their relative processing difficulties. For instance, the computational process of syntactic unification (Hagoort, 2005; Vosse \& Kempen, 2000) is more extended in time for sentences with non-adjacent compared to adjacent dependencies. This requires online processing memory, pointing to the close connection between memory resources and the processing of non-adjacent dependencies (Uddén, Ingvar, Hagoort, \& Petersson, 2012). The costs on memory and processing for non-adjacent dependencies is in line with evidence showing that these dependencies are mastered relatively late in infant development. For example, Gómez and Maye (2005) showed that while 15-month-old children were sensitive to a simple non-adjacent dependency, this was not the case for 12-month olds 
(for a recent review, see Folia, Uddén, De Vries, Forkstam, \& Petersson, 2011). Cotton-top tamarins (Newport, Hauser, Spaepen, \& Aslin, 2004), squirrel monkeys (Ravignani, Sonnweber, Stobbe, \& Fitch, 2013), and chimpanzees (Sonnweber, Ravignani, \& Fitch, 2015) are capable of acquiring simple non-adjacent dependencies, but work with these non-human primates has also shown greater processing difficulties when non-adjacent dependencies are directly compared with adjacent dependencies (Fitch \& Hauser, 2004).

Three FMRI studies suggest that the neural correlates of explicit non-adjacent dependency processing include Broca's region (Bahlmann, Schubotz, \& Friederici, 2008; Friederici, Bahlmann, Heim, Schubotz, \& Anwander, 2006; Makuuchi, Bahlmann, Anwander, \& Friederici, 2009). However, FMRI only characterizes the correlation between a cognitive task and the measured blood oxygenation level dependent (BOLD) signal. The observed activations might differ in their causal relation to task performance. In the present study we investigated two main questions: (1) whether there is a causal connection between activity in Broca's region and the processing of non-adjacent dependencies using repetitive transcranial magnetic stimulation (rTMS) in an artificial grammar learning paradigm; and (2) whether implicit processing of adjacent and non-adjacent dependencies requires the involvement of Broca's region. By implicit processing, we mean information processing resulting from implicit learning. Implicit learning is defined as "the process whereby a complex, rule-governed knowledge base is acquired, largely without any requirements of awareness of either the process or the product of acquisition" (Reber, Walkenfeld, \& Hernstadt, 1991). In particular, we note that implicit learning/processing happens in the absence of explicit strategies (e.g., problem solving). While the above FMRI studies are interesting as a first estimate of where non-adjacent dependencies are processed in an experimental setting with explicit instructions, we wanted to test whether these results could be extended to the more ecologically valid situation of implicit processing. We also investigate the potential causal connection between Broca's region and structured sequence processing in order to understand the functional organization of the inferior frontal cortex in general (Hagoort, 2005; Petersson et al., 2004; Uddén \& Bahlmann, 2012).

The causal role of Broca's region in language processing has been studied in the vast literature on Broca's aphasia, but there is still no consensus on whether Broca's region plays a causal role in syntactic comprehension. For instance, Dronkers (2000) argued that lesions restricted to Broca's area never lead to persistent Broca's aphasia and that damage of the tissue in the surrounding frontal cortex is necessary for more extensive symptoms than transient mutism. The grammaticality judgments of Broca's aphasics are often largely unaffected by the lesion, although sentence comprehension can be poor (Linebarger, Schwartz, \& Saffran, 1983). In addition to production impairments, Broca's aphasics are sometimes regarded as having a specific receptive syntactic disorder. What constitutes this receptive disorder remains an open question (Caramazza \& Zurif, 1976; Grodzinsky, 2000; Linebarger et al., 1983; Martin, Wetzel, Blossom-Stach, \& Feher, 1989). Testing causal brain function with TMS rather than lesions is advantageous since TMS combines systematic target locations with the absence of any adaptive changes as a reactive response to the lesion itself. The demonstration of a causal connection between Broca's region and implicit processing of non-adjacent dependencies would therefore be a step towards clarification of the exact nature of a potential receptive syntactic deficit caused by lesions or perturbation of Broca's region.

Having introduced the main motivation for the current study, we will specify the experimental paradigm and its relation to the neurobiology of language. Artificial grammar learning (AGL; see below for a concrete description of the task) is a well-established paradigm commonly used to investigate implicit structured sequence processing (Forkstam \& Petersson, 2005; Stadler \& Frensch, 1998). The implicit aspect of AGL makes it a suitable model for structural aspects of language acquisition in a laboratory setting (de Vries, Christiansen, \& Petersson, 2011). For example, the aspects of natural language syntax that are acquired as a consequence of supervised teaching are negligible compared to what is acquired spontaneously through exposure to well-formed examples, as in implicit AGL (Folia, Uddén, et al., 2011). Consequently, investigating the localization of implicit processing of non-adjacent dependencies increases the relevance of AGL results in relation to natural language. At the same time, we wanted to use the AGL paradigm because it provides a relatively uncontaminated window onto the neurobiology of structured sequence processing (e.g., no semantics in a linguistic sense) as an important aspect of syntax (Gomez \& Gerken, 2000; Petersson et al., 2004; Reber, 1967). There are examples of studies where the sentence-level semantics complicate the interpretation of FMRI results on the processing of non-adjacent dependencies (Makuuchi et al., 2009). Additional homologies between implicit AGL and natural language acquisition have been demonstrated during the course of development (Gerken, 2006; Gervain, Nespor, Mazuka, Horie, \& Mehler, 2008; Gómez \& Maye, 2005; Santelmann \& Jusczyk, 1998). Moreover, individual differences in non-adjacent dependency processing in natural language and statistical learning of non-adjacent dependencies in abstract sequences are correlated (Misyak, Christiansen, \& Tomblin, 2009). Together, this evidence suggest a strong link between natural language processing and implicit sequence learning (cf., Christiansen, Louise Kelly, Shillcock, \& Greenfield, 2010).

In this study we address the neurobiology of non-adjacent dependency processing in an AGL rTMS experiment. The studies, briefly outlined above, used considerably simpler grammars and less ecologically relevant paradigms, compared to the grammars and paradigm used in this study. Our paradigm improves on some of these aspects by: (1) using the largest artificial language size tested so far, both in terms of sequence lengths and the number of sequences participants were exposed to; (2) testing the most complex patterns investigated so far, with multiple non-adjacent dependencies with additional variance in pre- and postfix sequences with adjacent dependencies; (3) using an extended period of acquisition over several days in an implicit learning paradigm, which allows for natural abstraction and consolidation processes to take place (Nieuwenhuis, Folia, Forkstam, Jensen, \& Petersson, 2013); and (4) as in natural language acquisition, not instructing participants to extract regularities nor providing any type of performance feedback.

In the acquisition phase of AGL, participants are exposed to sequences generated from a complex rule system in a cover task. Participants are then instructed to classify novel items as grammatical, or not, based on their immediate intuitive impression, or guessing based on their "gut feeling". Gut feeling is referred to in order to minimize explicit problem solving strategies, which might lead to poor classification performance, for example, because irrelevant or incorrectly inferred rules are applied (Whitmarsh, Udden, Barendregt, \& Petersson, 2013). The AGL paradigm used in the present study is designed to test implicit processing (Folia \& Petersson, 2014). This is achieved by making the task substantially more difficult than is typically the case. Under this condition, explicit processing will typically be less successful than implicit processing. Furthermore, the types of violations we use exclude the possibility of successful classification based on explicit strategies such as counting, repetition monitoring, or equivalent (de Vries, Monaghan, Knecht, \& Zwitserlood, 2008). The acquisition phase was also considerably longer than in earlier studies of non-adjacent processing (Bahlmann et al., 2008; de Vries et al., 2008; Friederici et al., 2006) allowing consolidation and abstraction processes to take place over at least a week. Importantly, we measured implicit acquisition by adding an implicit test to the initial test phase, using a preference instruction (Forkstam, Elwér, Ingvar, \& Petersson, 2008). Here, participants are never informed about the existence of a grammar but 
are asked to make a preference choice (i.e., like/dislike) instead of making the standard grammaticality judgment. By not informing the participants about the existence of an underlying generative grammar, we minimize the motivation for the participant both to search for potential explicit strategies and using such strategies. The preference test is thus well-suited to assess implicit acquisition. When participants are subsequently informed of the presence of a grammar, this tends to boost the classification performance somewhat (Folia et al., 2008; Forkstam et al., 2008). These were the conditions under which the TMS stimulation was conducted in this study, and the participants were instructed, as in the preference test, to rely on their immediate gut feeling and avoid explicit problem solving to maximize their performance. At the very end of the experiment, we also probe the participants' potential explicit knowledge in a standardized post-experimental questionnaire.

In implicit AGL experiments, robust classification performance is accompanied by robust functional changes in language relevant brain regions, including Broca's region of the left inferior frontal gyrus (Folia, Forkstam, Ingvar, Hagoort, \& Petersson, 2011; Forkstam, Hagoort, Fernandez, Ingvar, \& Petersson, 2006; Lieberman, Chang, Chiao, Bookheimer, \& Knowlton, 2004; Petersson, Folia, \& Hagoort, 2010; Petersson et al., 2004). The inferior frontal region spans Brodmann's areas (BA) 44, 45 and 47 and functional neuroimaging results in AGL are typically centered on BA 44 and 45 . Moreover, the integrity of white matter tracts in the left, but not the right, inferior frontal region is associated with AGL performance (Floeel, de Vries, Scholz, Breitenstein, \& JohansenBerg, 2009). In a recent study, we demonstrated a causal role of Broca's region during classification with rTMS (Udden et al., 2008) consistent with previous FMRI results (Petersson et al., 2004, 2010). A causal role of Broca's region during acquisition has also been demonstrated with anodal transcranial direct current stimulation (de Vries, Barth, Knecht, Zwitserlood, \& Floeel, 2010). Moreover, language processing deficits are paralleled by impaired structured sequence learning/processing. This has been shown with agrammatic aphasics, predominantly with lesions in Broca's or the perisylvian language region (Christiansen et al., 2010; Hoen et al., 2003), traumatic brain injury patients with prefrontal damage (Pothos \& Wood, 2009), in individuals affected with specific language impairment (Evans, Saffran, \& Robe-Torres, 2009), dyslexia (for a review, see Folia et al., 2008), and adults with a history of speech and language problems (Richardson, Harris, Plante, \& Gerken, 2006). Together, these results provide strong support for the notion that Broca's region (BA 44/45) is causally involved in structured sequence processing (Udden et al., 2008) just as in natural language processing (Petersson \& Hagoort, 2012). However, this hypothesis also needs to be directly investigated in the healthy brain.

In this study, we investigate the hypothesis that artificial structured sequence processing after implicit acquisition is causally dependent on the activity in Broca's region using repetitive transcranial magnetic stimulation (rTMS). Previous FMRI studies that showed an involvement of Broca's region in non-adjacent dependency processing, have focused on explicit learning/processing (Bahlmann et al., 2008; Friederici et al., 2006; Makuuchi et al., 2009) which might or might not be relevant for natural language processing. Therefore, in implicit AGL, processing of non-adjacent dependencies has not yet been linked to activity in Broca's region. The alternative hypothesis is a more general role for Broca's region in structured sequence processing, independent of the nature of dependencies.

We used a design which improves on Uddén et al. (2009), who compared pre- and post-TMS classification. In the present study, we explicitly controlled potential time- or repetition effects by a balanced experimental design. We compare classification performance after stimulation of the left inferior frontal gyrus (LIFG; BA 44/45) and a non-language relevant control region (vertex) in an offline repetitive TMS paradigm. Thus, the current design con- trolled directly for both non-specific time effects as well as the unspecific effects of TMS itself.

\section{Methods}

\subsection{Participants}

Nineteen right-handed healthy university students volunteered to participate in the study (15 females, 4 males, mean age $\pm \mathrm{SD}=24 \pm 3$ years). They were all prescreened for relevant medical history, medication, drug abuse, head trauma, neurological or psychiatric illness, and family history of neurological or psychiatric illness, and had normal or corrected-to-normal vision. All participants gave written informed consent according to the Declaration of Helsinki and were explicitly informed that they could stop their participation at any time without having to give any explanation. The local medical ethics committee at the UMC St. Radboud approved the study. Four participants did not proceed to the TMS stimulation. Two of these participants changed their mind over their participation. Two participants did not engage in the task, so it was not meaningful to proceed with stimulation, since the prospect of acquiring meaningful data was too low (the criterion for this was reaching 60\% correct responses in the preference test). One participant was excluded from further analysis because a mistake was made when administering the task. One additional participant was excluded because this participant did not meet the inclusion criterion of not showing any signs of explicit strategy use, such as classification based on superficial stimulus features, including sequence length (the data indicated that this participant endorsed sequences based solely on sequence length). In the analysis of classification data before stimulation, all 19 participants were included, since the main purpose of this analysis was to replicate the behavioral results of successful acquisition of the complex non-adjacent dependencies (Uddén et al., 2009).

\subsection{Stimulus material}

We used a bounded (context-sensitive) grammar with crossed non-adjacent dependencies (see Fig. 2). Grammatical sequences were generated from a grammar with a crossed part. The sequence length was 6-12 symbols (mean sequence length 10.3 symbols). The part before and after the crossed part, which only included adjacent dependencies, used the alphabet $[\mathrm{M}, \mathrm{N}, \mathrm{V}, \mathrm{W}, \mathrm{T}, \mathrm{R}]$ of terminal symbols, while the crossed part used the alphabet $[\mathrm{F}, \mathrm{D}, \mathrm{X}, \mathrm{L}$, $\mathrm{P}, \mathrm{K}]$. The first half of the crossed part was always taken from the set $[\mathrm{F}, \mathrm{D}, \mathrm{X}]$ and the last half from [L, P, K]. These crossed parts were pre- and post-fixed with regular parts (see Fig. 1 for example sequences). This set-up dissociates the surface structure from the underlying grammar with the purpose of minimizing the usefulness of explicit memorizing or similar strategies.

Crossed dependencies were introduced as agreements between the letter pairs $F$ and $L, D$ and $P$, as well as $X$ and $K$, such that if there was an $F(D, X)$ in the first, second or third position of the first half, there was an $\mathrm{L}(\mathrm{P}, \mathrm{K})$ in the corresponding position in the last half of the sequence. There were 39 different correct crossed dependency fragments in the stimulus material. We constructed violations to these agreements by keeping the structure of F's, D's and X's in the first part and L's, P's, K's in the second part, but violating the agreements in the first, second, third position or in combination. We calculated the specific associative chunk strength (ACS, cf., Knowlton \& Squire, 1996; Meulemans \& Van der Linden, 1997) for each sequence in relation to the complete set of sequences. ACS is a measure of subsequence familiarity of an individual sequence in a test set, with respect to some acquisition set. To calculate ACS, the frequency of each possible bigram (two consecutive letters) and trigram in the acquisition set is first calculated. Then, each test sequence is decomposed into its bigrams and trigrams and its average bi- and tri- 


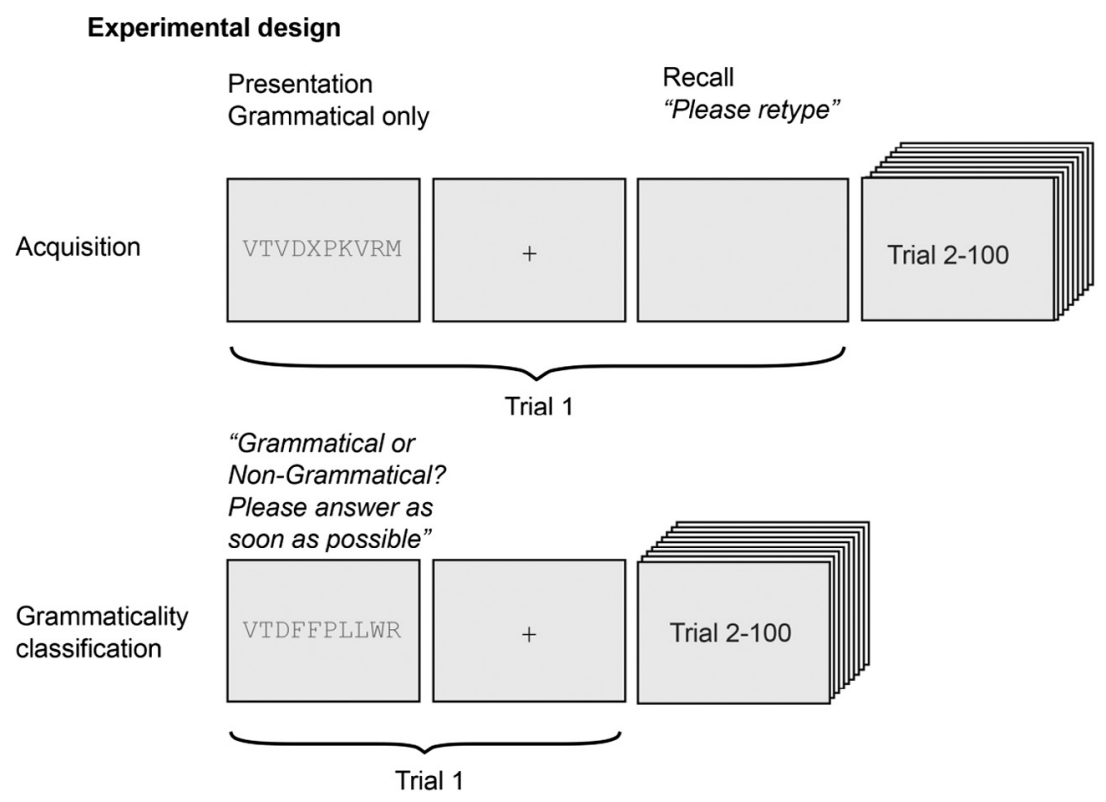

$50 \%$ Grammatical (not presented during acquisition) $50 \%$ Non-Grammatical

\section{Example stimuli}

\begin{tabular}{ll} 
Grammatical & Non-Grammatical \\
\hline VTVDXPKVRM & WSDXKPVRM \\
WTVDDPPWRN & VSXFPPVRM \\
WSDXDPKPVRN & VSXFFLPLVR \\
VTDFFPLLWR & WTWDXFKPLV
\end{tabular}

Fig. 1. Experimental procedures and example stimuli.

gram frequency is calculated. 100 sequences were randomly selected and tested with respect to its ACS content in order to generate an acquisition set which was representative in terms of ACS in comparison to the complete sequence set. The 5 classification sets were created in sizes of 100 sequences each (50 grammatical, 50 nongrammatical). Thus, each participant encountered 100 sequences in during acquisition and 100 different sequences during each classification session (i.e. they encountered 500 sequences during classification for the whole experiment). The non-grammatical sequences were derived from the grammatical sequences that were not used in the acquisition material. The test sets were selected so that for a given classification test set, the stimulus conditions did not differ significantly in terms of ACS or complete sequence length.

\subsection{Experimental procedure}

The complete experiment spanned nine days with one implicit acquisition session each day. On day one, a baseline preference classification was administered before the first acquisition session.

\section{Crossed}

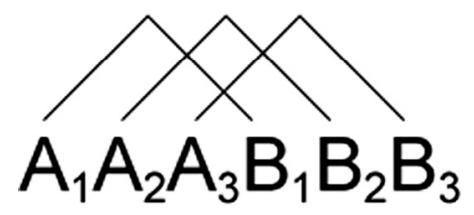

Fig. 2. In Experiment 1, we used a bounded crossed (context-sensitive) grammar. The non-adjacent dependencies are crossed in the manner indicated by the lines.
On day seven, participants performed a preference classification task after the acquisition session. Prior to the experimental TMS stimulation, we performed a motor threshold measurement to determine the individual stimulation intensity and acquire structural MRI data. The TMS stimulation was carried out on the 8th day, in a morning and an afternoon session separated by at least $4 \mathrm{~h}$. The participants were told about the existence of a grammar just before the TMS stimulation and performed the grammaticality classification task immediately after the TMS stimulation. On day 9, after their last acquisition session, participants performed a grammaticality classification task without stimulation. This was done on a separate day in order to assess the end-state of the implicit learning process independent of TMS. Of the 13 participants that were included in the TMS part of the study, seven participants started with vertex stimulation and six participants started with LIFG stimulation. Five participants responded "grammatical" with the right hand during vertex stimulation (and the rest responded "grammatical" with the left hand). Six participants responded "grammatical" with the right hand during LIFG stimulation. Thus, the counterbalancing of response hand and stimulation site was adequate.

\subsection{Implicit acquisition}

The acquisition task ( $\sim 30 \mathrm{~min})$ was presented as a short-term memory immediate recall task to the participants. During the acquisition task, each sequence was presented for $6 \mathrm{~s}$ (whole sequence presentation), centrally placed on a computer screen using Presentation (nbs.neuro-bs.com). After the sequence disappeared from the screen, participants recalled the sequence by self-paced typing on a keyboard. Participants were allowed to correct themselves but no perfor- 
mance feedback was provided. The participants were only exposed to grammatical examples and the presentation order of the 100 grammatical sequences was randomized over the acquisition sessions.

\subsection{Preference classification}

Participants were instructed to indicate if they liked a sequence or not, as fast as possible after sequence onset, based on their immediate intuitive impression (i.e., guessing based on "gut feeling"). They were told that there was no correct or incorrect response and that they should give one response per sequence based on their "gut feeling". The whole sequence was presented for $3.5 \mathrm{~s}$ followed by an inter stimulus interval of $2.5 \mathrm{~s}$, when a fixation cross was presented. The response hand was balanced across preference and grammaticality classification and across participants. Classification sequences were divided into separate sets and each participant was presented with each set of classification sequences once, either in the baseline preference task, the last preference task, or the grammaticality classification task. The presentation order of the classification sets was balanced across participants.

\subsection{Grammaticality classification}

After the last preference classification, on a subsequent separate day, the participants were informed about the existence of a complex set of rules used to generate the acquisition sequences (but they were not informed about the actual rules). They were then instructed to classify novel sequences as grammatical or not based on their immediate intuitive impression (i.e., guessing based on "gut feeling"). The participants were instructed to do this as correct and as fast as possible after sequence onset. Stimulus presentation parameters were the same as in the preference classification task.

\subsection{MRI data acquisition and TMS localization}

The stimulation site in the left inferior frontal cortex (BA 44/45; center of mass at MNI coordinates $[x, y, z]=[-48,16,20] \mathrm{mm}$, see Fig. 3) was selected based on the averaged overlap of the left inferior frontal activation observed in two FMRI studies of processing of artificial syntax (Forkstam et al., 2006; Petersson et al., 2004). The target and TMS protocol (20 min $1 \mathrm{~Hz}$ repetitive TMS at $110 \%$ of resting motor threshold) was the same as in the TMS-experiment on grammars with adjacent dependencies (Udden et al., 2008). The marked participant-specific MRI images were co-registered with the TMS coil and the participant's head using the BrainSight Frameless Stereotactic System (Rogue Research, Montreal, Canada). During the delivery of the rTMS pulses, the 3D position of the coil was continuously monitored. The motor threshold was defined as the intensity that evoked a motor potential of at least $50 \mu \mathrm{V}$ in the first dorsal interosseus muscle in at least 5 out of 10 pulses. This level of stimulation was well-tolerated by all participants and the concurrent stimulation of facial muscles and the trigeminal nerve was limited and mainly related to the masseter muscle. Repetitive TMS was delivered through a hand-held focal Figure-8-shaped coil with a $70 \mathrm{~mm}$ wing diameter (Magstim Co., Whitland, Dyfed, UK) held tangentially to the scalp with the focal point of the coil as close as possible to the marked target position. The induced current direction was held constant over the stimulation period and over participants through orientation of the coil handle in a medial-posterior direction in relation to the vertex, with approximately $45 \mathrm{deg}$ angle to both the central sulcus and the inter-hemispheric longitudinal fissure. For the control stimulation of the vertex, the center of the coil was held over the vertex and the handle pointed backwards, in line with the interhemispheric longitudinal fissure and with 90 deg angle to the central sulcus. Vertex was localized as the point midway between the inion and the nasion, equidistant from the left and right ear.

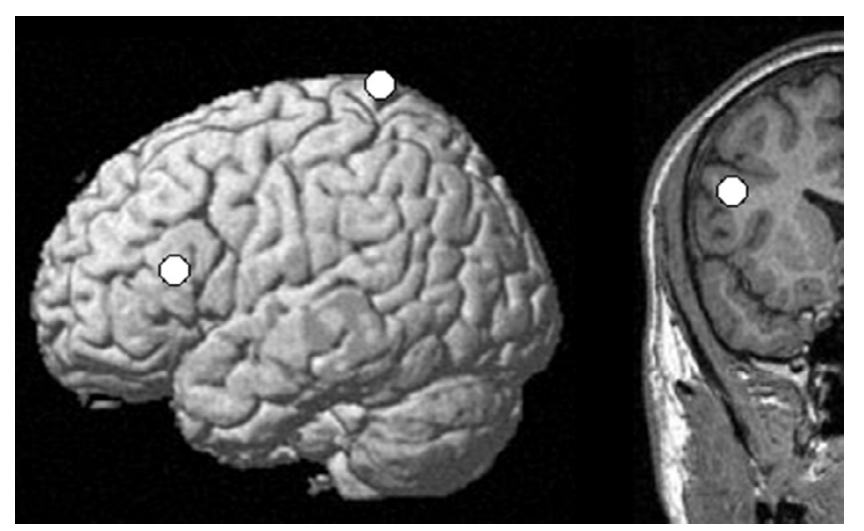

Fig. 3. TMS target sites. Left: the left inferior frontal target region (BA 44/45) and the non-language relevant control region (vertex). Right: the left inferior frontal stimulation site shown on a coronal slice.

\subsection{Data analysis}

Repeated-measures ANOVAs and $t$-tests were used to analyze the data and a significance level of $p<0.05$ was used throughout the study. We analyzed the classification performance with endorsement rate as the dependent variable. The endorsement rate is defined as the number of sequences classified as grammatical independent of their actual status, divided by the total number of recorded responses for each factor level (Meulemans \& Van der Linden, 1997). When analyzing the behavioral accuracy results from the preference tests, the first factor was TEST, with two levels corresponding to the baseline preference tests and the last preference test at day seven. The second factor GRAMMATICALITY had two levels: grammatical and nongrammatical sequences. This factor was also used when analyzing the results from the grammaticality classification on the last day, where we compared the classification performance to chance. When analyzing the data from the TMS sessions, we used the same factors as above, but the TEST factor was exchanged for the factor TMS, which had two levels: LIFG and VERTEX. Sizes of effects are reported as differences between means and goodness of fit is reported using the T or F-statistic or Cohen's d (Lenhard \& Lenhard, 2016). Mean values are reported with the standard error of mean.

We also analyzed response times, calculated from the presentation onset until the time of response. The response time analysis was performed on correctly classified sequences only. The few response times faster than $400 \mathrm{~ms}$ were not analyzed (in total 5 trials or less than $0.1 \%$ ). The next sequence was presented $6 \mathrm{~s}$ after the previous sequence onset. The response times obtained from each trial were analyzed in a mixed linear model (Field, 2009) with participants as random factor and the factors GRAMMATICALITY and TEST (defined as in the previous section) as fixed factors. This means that the average of each participant will be estimated, while the distributional information in the response times is not thrown away. We also tested response times in the last day grammaticality test in a second mixed linear model. This was done in order to test whether we could detect response time differences between the conditions in the end-state of learning, where we expected highest sensitivity to the grammaticality factor (i.e., difference in endorsement rates between grammatical and non-grammatical sequences). We rounded the resulting degrees of freedom to integers.

\section{Results}

\subsection{Classification without TMS}

The acquisition effect was analyzed by comparing the preference classification on the seventh day with the baseline preference classification. We used a two-tailed paired $t$-test, with the differ- 
ence in endorsement rates between grammatical and nongrammatical items as the dependent variable. This test was significant $(T(18)=2.73, p=0.01)$ confirming successful acquisition of significant aspects of the underlying grammar. This replicates previous findings (Uddén et al., 2009). In the baseline preference session, the mean endorsement rate for the grammatical sequences was $0.48 \pm 0.03$ and for violations $0.46 \pm 0.03$. In the last preference session the mean endorsement rate for the grammatical sequences was $0.60 \pm 0.03$ and for violations $0.46 \pm 0.03$ (mean difference $0.14 \pm 0.03$ ). The average response time during the last preference session was $2.84 \pm 0.04 \mathrm{~s}$. We also analyzed the last grammaticality classification session against chance performance and found that participants were significantly better than chance $(T(14)=4.71$, $p<0.001$ ), consistent with previous findings (Uddén et al., 2009). In the grammaticality session, the mean endorsement rate for the grammatical sequences was $0.66 \pm 0.04$ and for violations $0.38 \pm 0.05$ (mean difference $0.28 \pm 0.05$ ).

\subsection{Classification with TMS}

The main effect of TMS was not significant $(p=0.36)$. This means that there was no shift in bias due to TMS. The GRAMMATICALITY factor was significant $(F(1,12)=41.31, p<0.001$, Cohen's $d=1.98)$. The interaction between TMS and GRAMMATICALITY was significant $(F(1,12)=7.18, p<0.05$, Cohen's $d=0.61$, see Fig. 4$)$, which reflected a greater endorsement of grammatical sequences after the control stimulation compared to the target LIFG stimulation. Thus, perturbation of the LIFG causes interference with syntactic classification. The effect of TMS for grammatical sequences was significant $(F(1,12)=6.50, p<0.05$, Cohen's $d=0.55)$, while the corresponding effect for non-grammatical sequences was nonsignificant $(F(1,12)=0.31, p=0.59$, Cohen's $d=0.12)$. The average endorsement rates for LIFG grammatical sequences were $0.63 \pm 0.04$, LIFG violation sequences $0.43 \pm 0.05$, vertex grammatical sequences $0.70 \pm 0.02$, vertex violation sequences $0.41 \pm 0.04$. The mean difference between grammatical sequences and violation sequences was $0.24 \pm 0.04$ and the mean difference between the grammaticality effect for LIFG compared to vertex was $0.18 \pm 0.07$.

The average response time during grammaticality classification was $2.85 \pm 0.03 \mathrm{~s}$. The response times were not modulated by TMS $(p=0.43)$ and the interaction between GRAMMATICALITY and TMS was not significant $(p=0.26)$. This speaks against a speed-accuracy trade-off as an explanation for the TMS results.

\subsection{Post-experimental questionnaire}

The post-experimental questionnaire was distributed after the last grammaticality test. Participant reports were, in our experience, typical for AGL. Since we had informed them of the presence of a grammar, it was not surprising that most participants recognized that there were some sort of dependencies in the sequences. However, when participants were given a list of correct and incorrect statements about the grammar and were asked to indicate whether they thought they were correct/incorrect and whether they used any of these during classification, only two participants thought that the full rule producing all crossed parts was true, and these two as well as an additional participant claimed to have used some of this knowledge during classification. These three participants did not show any performance differences compared to the rest of the participants and their TMS effect showed the same pattern as the rest of the group, as indicated by a non-significant interaction between the TMS and GRAMMATICALITY interaction and the between participant factor separating these three participants $(p=0.29)$. In other words, self-reported insight did not translate into a performance benefit or a different TMS effect. In fact, the low recognition rate of the correct rules suggests that participants

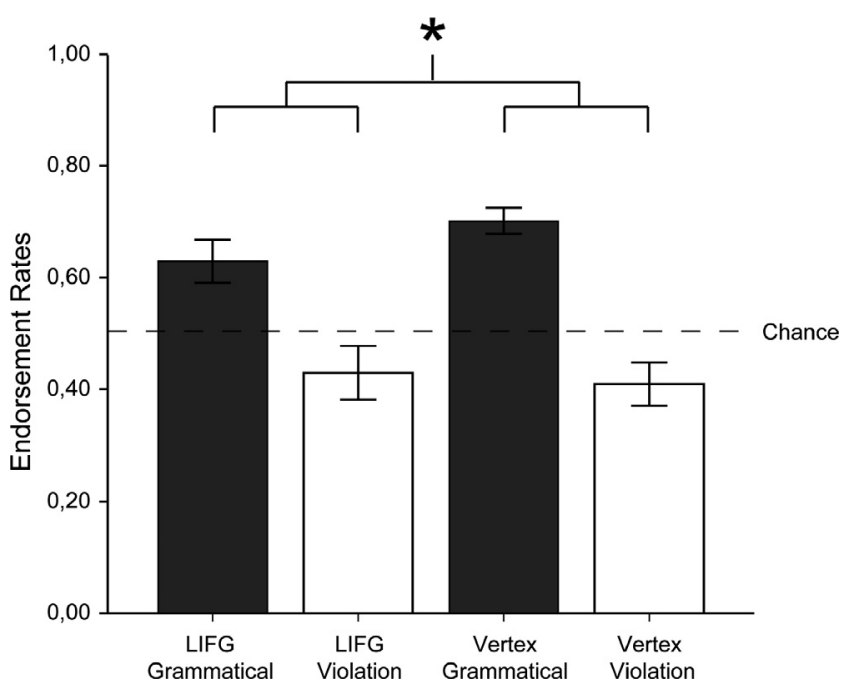

Fig. 4. The endorsement rates of grammatical (black) sequences and sequences with violations (white) in the two classification test with stimulation of either the left inferior frontal gyrus (LIFG) or vertex. TMS to the LIFG led to significantly impaired classification performance compared to control stimulation at vertex, seen here as a smaller separation in the endorsement rates between grammatical and violation sequences to the left compared to the right. Error bars indicate the standard error of the mean.

had little or no explicit knowledge of the underlying grammar. Likewise, there is little or no evidence for the use of any explicit strategies during classification. At the group level, the participants classified the correctness of the stated rules at guessing level.

\section{Discussion}

The main result of this study provides support for a causal role of the left inferior frontal gyrus (LIFG; BA 44/45) in the processing of sequences with crossed non-adjacent dependencies. This emphasizes the importance of Broca's region as a node in the brain network that process structured sequences such as those generated by artificial and natural grammars. Syntax processing deficits can be found in the absence of lesions to Broca's region, and conversely, lesions of Broca's region are often found without persistent concordant syntactic deficits (Dronkers, Wilkins, Van Valin, Redfern, \& Jaeger, 1994), sometimes in as many as $50 \%$ of the cases (Dronkers, 2000). Our results cast new light on these results. For instance, it is possible that these patients would be impaired at artificial grammar learning, where they cannot rely on semantic information (see Caramazza \& Zurif, 1976), or where they have to process newly or recently acquired sequence structures. Indeed, there is evidence suggesting impaired sequence processing after lesions to this region (Christiansen et al., 2010; Hoen et al., 2003; Pothos \& Wood, 2009). Our demonstration of a causal connection between activity in Broca's region and grammaticality judgments in structured sequence processing is important because grammaticality judgments are often spared in Broca's aphasics who at the same time display impaired sentence processing (Linebarger et al., 1983).

Explicit acquisition (for further specification, see de Vries et al., 2008) of non-adjacent dependencies (the $A^{n} B^{n}$ grammar) has been reported (Bahlmann et al., 2008; Friederici et al., 2006). These FMRI studies, using explicit paradigms in which the participants were instructed to extract the rule behind the acquisition sequences during acquisition, reported effects of non-adjacency processing in Broca's region, in line with current findings.

Taken together, the results of the current study, which show a causal role for Broca's region in processing crossed non-adjacent dependencies, together with previous results showing a causal role for Broca's region in processing adjacent dependencies (Udden et al., 2008), suggest that Broca's region is engaged in structured 
sequence processing independent of adjacency type (Petersson et al., 2010). Our results are also informative in relation to theories on processing of center-embedded structures in Broca's region. In the AGL literature, sequences are often informally described as hierarchical if they involve multiple levels of nested structures (exemplified by multiple center-embeddings). Some authors have claimed that Broca's region is specifically involved in the processing nested ('hierarchical') sequences (Koechlin \& Jubault, 2006) and others have demonstrated additional involvement of Broca's region when processing nested structures, compared to adjacent dependencies, using FMRI (Friederici et al., 2006; Opitz \& Friederici, 2007). Here, we provide evidence that, in addition to adjacent dependencies (Udden et al., 2008), also crossed structures are processed in Broca's region.

No stimulus material, including in this study, can control for every conceivable aspect of sequence similarity or way in which the classification sequences might resemble acquisition sequences, and therefore might potentially have contributed to observed performance. There is some evidence that some such sequence aspects contribute to classification performance irrespective of grammaticality (see review in Kinder \& Lotz, 2009, as well as Opitz \& Hofmann, 2015; Pothos \& Bailey, 2000). For instance, we did not control for so-called repetition structures, as defined by Brooks and Vokey (1991). For example, 'FFDLLP' and 'DDFPPL' may conceivably be represented abstractly as '112334', were the latter is supposed to capture the structure of repetition of certain elements. However, in our study grammatical and non-grammatical sequences were classified differently and we controlled for one important aspect of similarity with the ACS measure. In addition, and in the light of subsequent experiments in which we explored the generalization capacity of participants, it is clear that the acquisition effect is not captured by repetition structures.

In this study, we demonstrate a causal connection between Broca's region and the processing of artificial grammars with crossed non-adjacent dependencies. Broca's region plays a role in language processing, in particular in the syntactic domain (Segaert, Menenti, Weber, Petersson, \& Hagoort, 2011). The posterior part of the left inferior frontal region, including BA 6, is also involved in structured sequence processing in the phonological domain (Cristià, Seidl, \&
Gerken, 2011; Tessier, 2007). The left inferior frontal region has also been implicated in processing of language relevant gesture information (Straube et al., 2013; Willems \& Hagoort, 2007), sign-language (Courtin et al., 2011), as well as the processing of structured sequences in music (Cristià et al., 2011; Janata \& Grafton, 2003; Maess, Koelsch, Gunter, \& Friederici, 2001; Patel, 2003; Patel, Iversen, Wassenaar, \& Hagoort, 2008; Tessier, 2007; Tillmann et al., 2006). For a review of perceptual, cognitive or action sequences that has been related to Broca's region see Christiansen et al. (2010). Taken together, this suggests a role for Broca's region in general structured sequence processing, whether the dependencies are adjacent or non-adjacent (for FMRI-results supporting this notion, as well as further discussion on this issue, see Petersson et al., 2010).

\section{Conclusion}

In this study, we show that rTMS applied to Broca's region interferes with classification of sequences with multiple crossed nonadjacent dependencies. Overall, the results support the view that Broca's region is engaged in structured sequence processing and provide evidence for a causal role of Broca's region in syntax processing.

\section{Acknowledgement}

This work was supported by the Max Planck Institute for Psycholinguistics, Donders Institute for Brain, Cognition and Behaviour, Centre for Cognitive Neuroimaging, Radboud University Nijmegen, Fundação para a Ciência e Tecnologia (PTDC/PSI-PCO/110734/2009; IBB/CBME, LA, FEDER/POCI 2010; PEst-OE/EQB/LA0023/2013), Vetenskapsrådet (8276), and The Swedish Dyslexia Foundation. We also want to thank Dr Gijs van Elswijk, Moniek Munneke and Dr Sebastiaan Overeem for their help in the TMS laboratory and with the ethics proposal.

\section{Appendix A}

Flow chart on stimulus creation

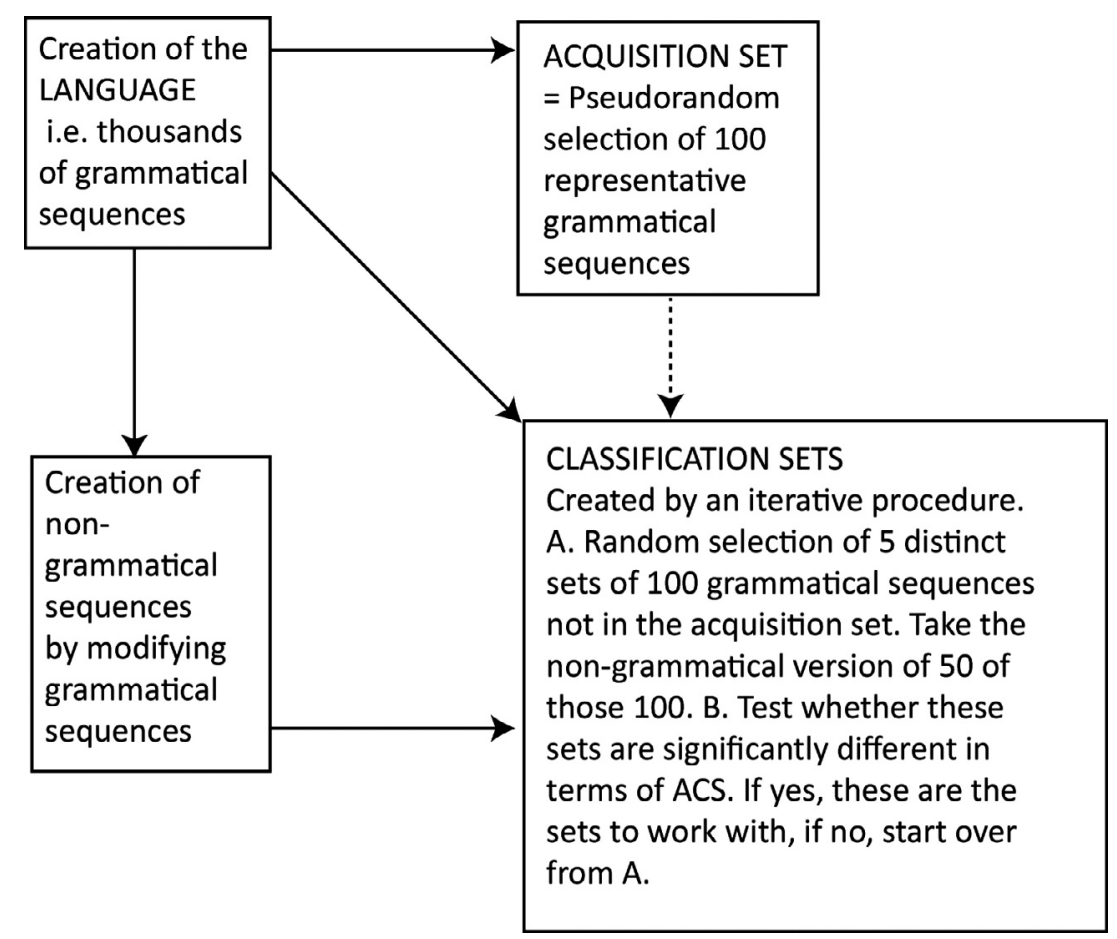


Acquisition set

\begin{tabular}{|c|c|c|}
\hline VTXFKLVS & VTXFXKLKVR & WTDXPKWS \\
\hline VTWFDDLPPVRN & MFXLKWRM & VTDXFPKLVRM \\
\hline NXDKPV & VTWFDFLPLVRM & VTXDDKPPVRN \\
\hline WTVDXDPKPWS & VSXKWRN & WTWFXLKV \\
\hline VTFDFLPLW & VTXFKLWRM & \\
\hline WSXXDKKPVRM & VTVXDKPW & \\
\hline NFFLLWR & VTWXFKLVR & \\
\hline WTXDKPW & VTXXFKKLVR & \\
\hline VSXFKLWS & VTVFXLKVR & \\
\hline WSFFFLLLWR & WTXFKLV & \\
\hline VTVXXDKKPV & WSXFKLVS & \\
\hline WTWDPWS & MXXXKKKWRN & \\
\hline WTWDDFPPLVS & WTDDPPVRN & \\
\hline VSXXFKKLVS & MXKW & \\
\hline VSXFKLWS & VTVDXFPKLWS & \\
\hline VTWFDFLPLWR & NXXKKVR & \\
\hline WSFDFLPLW & WSFXFLKLWRM & \\
\hline WTDDPPVRN & NFFFLLLVR & \\
\hline WSDFPLWRM & VTWFDFLPLWRM & \\
\hline NXKWRN & WTWXDXKPKVRN & \\
\hline VTXXDKKPVRM & WTXFDKLPVS & \\
\hline VSXDFKPLWRN & NDDDPPPVS & \\
\hline VTWXXXKKKVR & WTFXLKWRM & \\
\hline NFDLPWRM & VSDFDPLPVR & \\
\hline MFXLKWRM & WTVFFFLLLVR & \\
\hline VSDXPKWS & VSDXPKWRM & \\
\hline WTDXPKWRM & VTWDXPKW & \\
\hline VTVDFPLVR & WTWFFDLLPVRN & \\
\hline VTWFFLLWS & MXFKLWR & \\
\hline NFFLLW & NDXDPKPWRN & \\
\hline VTWXKVR & VSDXDPKPVRM & \\
\hline WSFFFLLLWR & VSXKVS & \\
\hline VTWDDPPWRN & WTVFDFLPLWR & \\
\hline VTWDFFPLLWRN & WTWDFPLVRM & \\
\hline WTWFFFLLLVS & WSDXFPKLVS & \\
\hline WTVFDLPVR & VTWDFFPLLWRN & \\
\hline VTXXKKW & WTWDFPLVRM & \\
\hline WTDFFPLLVS & WTWXDXKPKVR & \\
\hline VTWFXLKVS & WTVDDPPW & \\
\hline WTWDFDPLPWRN & WTWXFDKLPWR & \\
\hline WSFDLPW & WSXXKKWS & \\
\hline WTVDXFPKLW & VSDFPLVRN & \\
\hline WSXFKLWRM & MXDKPVRN & \\
\hline WTWDDPPVR & WTVDXFPKLWR & \\
\hline WTVXXXKKKVS & WSDPWR & \\
\hline MFXDLKPWS & NDDPPWS & \\
\hline VTVDPWRN & VTFXLKVR & \\
\hline NFXDLKPVRM & VSFFDLLPVRN & \\
\hline
\end{tabular}

\section{Classification set 1}

\begin{tabular}{ll}
\hline Grammatical & Non-grammatical \\
\hline VTVDXPKVRM & WTVXDKLWS \\
VTVFDLPWRM & VTVDXKLVS \\
VTVXDKPWRN & VTVXXKLVR \\
VTVDDPPWRN & WTVDFPKVR \\
VTVDDPPWRM & VTVDFKLVS \\
WTVDDPPWRN & WSDDKLWRN \\
WTVDDPPWRM & WSDXKPVRM \\
VTVFDLPVRN & VTVXDPLWR
\end{tabular}

Appendix A (continued)

\begin{tabular}{|c|c|}
\hline Grammatical & Non-grammatical \\
\hline WTVFDLPVRN & VSDXKLVRM \\
\hline WTWFXLKWR & VTWXXKLVS \\
\hline VTVXDKPVRN & VSFDPLWRN \\
\hline WSFFLLVRN & VSXFPPVRM \\
\hline VTVXFKLWS & WSXFLLVRN \\
\hline WTWXXKKVRN & WSFFLPVRM \\
\hline VTVDDPPVRM & WTWXDKLVS \\
\hline WTWFXLKVR & VTXFPKVRM \\
\hline VTWFXLKVR & VTWFXKLWS \\
\hline WTVXFKLWS & VTVFXKLVRM \\
\hline WTWDXPKWR & WTVFXKLVRN \\
\hline WSXDKPVRN & WTVXXKLVRM \\
\hline WTVFFLLWR & VTVFFLPVRM \\
\hline WTWDDPPWR & VTVDXKPVRM \\
\hline VSFFLLWRN & VTVDXKPVRN \\
\hline VTWFDLPWR & WTVDDKLWRM \\
\hline VTWDDPPWR & VTVXDKLWRN \\
\hline WTWDDFPPLWS & NFXFKLPVRM \\
\hline WTDFDPLPVR & VTVDXFLPPVRM \\
\hline WSDXDPKPVRN & VTDDDPKLWRN \\
\hline WSXFFKLLWR & NFDFPLPVRM \\
\hline WSXDFKPLWRM & VTVDXFKLKWRN \\
\hline VTDXDPKPVRM & VSXDFPLPVR \\
\hline WTDXDPKPVR & VTXDXKLPVRM \\
\hline VTDFFPLLWR & VTXFFLKLVR \\
\hline WSXXFKKLWR & WSFDFPKLVR \\
\hline WSFDFLPLVR & VTVDXFKKLVR \\
\hline WTWFFDLLPVR & WTVFXFKLPVRM \\
\hline VTWXDFKPLVRM & VSXFFLLPVRN \\
\hline WTDFFPLLWR & VTXFXKLPVRN \\
\hline WTWDDFPPLVR & VSXFFLPLVR \\
\hline VTDFFPLLWRN & WTWDXFKPLV \\
\hline VTWXFFKLLVRM & WTDDDPKLWRM \\
\hline MDXFPKLWRN & MXFFLPLWRN \\
\hline VTXXFKKLVRM & VTVXDDPKLWRN \\
\hline VTXXFKKLVRN & VTXFDPPPVRM \\
\hline WSXXFKKLWRM & VSXDFLPLVR \\
\hline WTWDXDPKPWRM & WSFDDPKLWRN \\
\hline WTXFFKLLWRN & MDXFKLLWRN \\
\hline VTXFDKLPVRN & VTVDXFKPLVRM \\
\hline VTWXXFKKLVR & VSXFXKKLWRM \\
\hline VTVDXFPKLVR & VTVDFFLPLVRM \\
\hline
\end{tabular}

\section{Classification set 2}

\begin{tabular}{ll}
\hline Grammatical & Non-grammatical \\
\hline VTVFDLPWRN & WTVDDPLVS \\
WTVDXPKVRM & VSXFLKVRN \\
WTVDXPKVRN & VTVDXKLWS \\
VTVXDKPWRM & WTVDXPLVR \\
VTVDXPKWRN & WTXFLKVRM \\
VTVDXPKWRM & VSFXKLWRN \\
WTVDXPKWRN & VSFXKLWRM \\
VTVFDLPVRN & WSXXKLVRM \\
VTVFFLLVRN & WSFXKLWRN \\
WTWFXLKWR & VTVDXKPVR \\
VTVXDKPVRN & WSXDKLVRN \\
WSFFLLVRN & VTFXKLWRN \\
VTWFXLKWR & WSDDPLWRN
\end{tabular}

(continued on next page) 
Appendix A (continued)

\begin{tabular}{ll}
\hline Grammatical & Non-grammatical \\
\hline VTWXXKKVRM & VSXFPPVRN \\
WTWDXPKVR & WTDXKLVRM \\
WTVDFPLWS & VTVDDPLWR \\
WTVXFKLWS & WTFDPLWRM \\
VTXDKPWRN & VTVFXKLVR \\
WSXDKPVRM & WTVFXKPVRN \\
VTWDXPKWR & WTVXXKLVRN \\
VTWXDKPWR & VTVFXKLWRM \\
WTWFDLPWR & VTVDXKLVRM \\
VSFFLLWRN & WTVFFLPVRN \\
VTDFPLVRM & VTVFDPLWRM \\
WTFFLLWRN & WTVXXKLWRN \\
WTXXDKKPVRN & VSFXFKLPVRM \\
WTXFFKLLVRM & VTWFDFLKPV \\
WTVXFFKLLWRM & WTVFDDPKLWR \\
VTWDFDPLPW & WSXFDPPLWR \\
WTWXFDKLPWR & WTVDXFLLLRM \\
VTXFDKLPWRN & WTXFFLKPVRN \\
VTWFFFLLLV & WSXDXKKLWRM \\
WTDXFPKLWS & VTVDXDPKLVRM \\
NFDFLPLVRN & VTVDFFLPLVR \\
WTWXDFKPLVRM & VTFXDPKLWRM \\
WTXFDKLPVRM & VTXFFLPLWS \\
WSDXFPKLVR & VSXDFLPLVRM \\
VTWXXDKKPVRM & WTVXFFLPLWR \\
WSFFFLLLWRM & WTDFFLPLVR \\
VTWFFDLLPVR & VTXFFLLVR \\
MFDFLPLWR & WTVDXFKKPVR \\
WTWFXFLKLVR & NDXFKLPVRN \\
VTWDDFPPLVR & NDFDPKLWRM \\
WSDXFPKLVRM & WTWFXDPKLVS \\
VTXFDKLPVRM & WTVXDDPKLWRN \\
VTWDDFPPLVRN & VTFDDPKLWRN \\
WTDFFPLLWRN & VTVDXDPKLVRN \\
VTXFFKLLWR & WTVFDDPKLWRN \\
WTDFFPLLWRM & VSXDFLKLWRM \\
WTXFFKLLWRM & \\
\hline & \\
&
\end{tabular}

\section{Classification set 3}

\begin{tabular}{ll}
\hline Grammatical & Non-grammatical \\
\hline VTVDXPKVRN & VTVDDPLWS \\
WTVFDLPWRN & WTVXFPLVS \\
WTVFDLPWRM & WSFXKLVRN \\
VTVDDPPWRM & VTWXXKPVS \\
WTVDDPPWRN & VTVDFLLWS \\
WTVXDKPWRM & VTXFLKVRM \\
WTVDXPKWRM & WSDXKLVRN \\
VTVFFLLVRM & WTVDDKLWR \\
VTVXDKPVRM & WSXFPPVRM \\
VTWXDKPVS & WSXDKLVRM \\
WTWXXKKVRM & WSXFPPVRN \\
VTWFXLKWR & VTVFFKLWR \\
WTXDKPWRM & WTVDXKLVR \\
WTWXXKKVRN & VTFXKLWRM \\
WTWDDPPVS & WTVDFLLVR \\
WTVDFPLWS & WSFDKLWRN \\
VTWFXLKVR & VTVDFKLVR \\
WTDDPPVRM & VTVFXKPVRN \\
VTXDKPWRM & VTVXXKLVRM
\end{tabular}

Appendix A (continued)

\begin{tabular}{ll}
\hline Grammatical & Non-grammatical \\
\hline VSXDKPVRM & VTVFFKLVRM \\
WTVFFLLWR & WTVFXKLWRN \\
WTWDDPPWR & VTVXFKPVRN \\
WSDFPLVRM & WTVDXKLVRM \\
WSDFPLVRN & WTVDFLLVRM \\
VTDFPLVRM & WTVFDPLWRN \\
WTFFFLLLR & MXFDPKLWR \\
WSXXFKKLVRM & NFXFKLPVRN \\
VTWXDDKPPVRN & WSXXXKKLWRM \\
VTWDFDPLPW & NFFDPKLWRM \\
WTVDFDPLPVR & WTFFDPKLWRM \\
VTWDXDPKPVS & WSFDFLLPVRN \\
WTWXDXKPKWRM & VSXDDPKLWR \\
WTFFFLLWRM & WTVFXDPKLWRM \\
WTXDFKPLWRN & WTFDDPKLWRM \\
VTWXFDKLPWRM & VSXFFLLPVRM \\
WTWDXDPKPWR & VTVFDDPKLWRM \\
WTWXXDKKPVRN & WTXFFLPPVRN \\
VTWDDDPPPVRM & VSXFXKKLWRN \\
WTFDFLPLVRM & VTDFDPKLWR \\
WSDFFPLLWRM & WTXFDPKPVR \\
VTWXDFKPLVRN & VTXFFLKPVRM \\
WTFDFLPLVRN & WSXXFKLPVR \\
WTFDFLPLVRN & WSDXFPKPVR \\
VSDFFPLLWRM & WSFDFLLPVRM \\
VTWDDFPPLVRM & WTVXXDPKLWRN \\
VTWDXDPKPWRN & WTVFFDPKLWRM \\
WTWXXFKKLVR & VTWDXFKLKWS \\
VSXXFKKLWRN & WTVDXFKKLVR \\
VSDXFPKLVRN & VTXFDPPLVRM \\
NDXFPKLWRN & WTWDXFKKLV \\
\hline & \\
\hline
\end{tabular}

Classification set 4

\begin{tabular}{ll}
\hline Grammatical & Non-grammatical \\
\hline VTVFDLPWRM & WTVFFKLWS \\
WTVDXPKVRM & VTVDFLLVS \\
WTVFDLPWRM & VTVXXKPVR \\
VTVDXPKWRN & VTWXFKKWS \\
VTVDXPKWRM & WTVDFKLVS \\
WTVDXPKWRN & VTXFLKVRN \\
VTVFDLPVRM & WTVDFPPVR \\
VTVFFLLVRM & VTVXFKPVR \\
WTVFFLLVRM & VTVDXKLVR \\
VTVDFPLWS & VTDDKLWRN \\
VTDDPPVRN & VTDFLLVRM \\
VTVXFKLWS & WTVDXKPVR \\
VTWXXKKVRM & VSXXKLVRN \\
VTVDDPPVRM & WTXFLPWRN \\
WTWDDPPVS & VSXFLLVRM \\
VTWDXPKVR & WSFFLPVRN \\
VTWDXPKVR & WTVDDPLWR \\
WTDDPPVRM & WTVFXKLVRM \\
VTVFFLLWR & VTVDFPKVRN \\
WTWXDKPWR & WTVXXKPVRN \\
VSXDKPVRN & VTVFFLPVRN \\
WTWFDLPWR & VTVDXKLVRN \\
WTFFLLWRN & WTVDXKPVRM \\
VSFFLLWRM & WTVDXKLVRN \\
VSDFPLVRM & WTVDFLLVRN \\
\hline
\end{tabular}


Appendix A (continued)

\begin{tabular}{ll}
\hline Grammatical & Non-grammatical \\
\hline WTWDFDPLPW & VSXFFLLLRM \\
WSXDFKPLWRN & MFXFKLPVRN \\
WTVDDFPPLWRN & WTFFDPKLWRN \\
VTWFXFLKLWS & VTVXXDPKLWRM \\
VTXDFKPLVRN & NFDFPLPVRN \\
NDXFPKLVRM & VTFFDPKLWRM \\
VTWDFDPLPWS & WTWFXDPKLW \\
WTWXFFKLLVR & WTWDXFKLKV \\
VSFDFLPLVRN & VSXFDPKPVRN \\
WTVXXFKKLWRM & WTVDXFLLPVR \\
WTVFFFLLLWRM & WTVDXFKPPVRN \\
WTDFFPLLWR & WTXFFLLLVRN \\
WTXFFKLLWR & VSXDFPLPRM \\
WTXFDKLPVRN & NXDFLPLWRN \\
VTWDDDPPPVRN & VTXXXKKLWR \\
WTWFFFLLLW & VTVDXFLPLVR \\
VSDFFPLLWRN & WSDXFPLPVRM \\
VTWFFFLLLW & VSDFDLPLWR \\
VTXDFKPLWRM & WSFXDPKLWRM \\
WTWDXDPKPWRN & VTVDXFKPPVRN \\
VTWFXFLKLVRM & WTVDDDPKLWRN \\
VSFDFLPLWR & VTFDDPKLWRM \\
WTWXDFKPLWR & VSDFFLPLVRN \\
VSDXFPKLVRN & VSDXFLPLVRN \\
WTWXXFKKLVRM & MXDFLPLWRN \\
\hline
\end{tabular}

\section{Classification set 5}

\begin{tabular}{ll}
\hline Grammatical & Non-grammatical \\
\hline VTVDXPKVRN & WTVDFLPWS \\
WTVDXPKVRN & WTVDXKLVS \\
VTVDDPPWRN & WTVDFLLVS \\
WTVXDKPWRN & WTVDXKLWS \\
WTVXDKPWRM & WTVDFLLWS \\
WTVDDPPWRM & WSDXKLVRM \\
VTVFDLPVRM & VTWDFLKWS \\
WTVFDLPVRM & WTWXXKLVS \\
WTVFFLLVRM & VTVFDPLWR \\
WTVFFLLVRN & WSFXKLWRM \\
WTVDDPPVR & VTVXDKLWR \\
VTWFDLPVS & VTWDFPKVS \\
WTVXDKPVRM & VTWXXKLWS \\
WTVXDKPVRM & WTVXDKLWR \\
WTWFXLKVR & WTFDPLWRN \\
VTWDDPPVS & WSFDPLWRN \\
VTWDDPPVS & WTXXKLVRN \\
WSXDKPVRM & WTVFXLWRM \\
VTVFFLLWR & VTVXXKPVRM \\
WTWXDKPWR & VTVDFPPVRN \\
VTWXDKPWR & WTVFXKLWRM \\
WSDFPLVRM & VTVDFLLVRM \\
VTWDDPPWR & WTVXFPLVRN \\
VSFFLLWRM & WTVDXKPVRN \\
VTVXFKLVR & VTVDFKLVRM \\
NXXFKKLWRN & NFDFPKPVRN \\
VTDFDPLPVRN & VTXFDPKLW \\
VTVDFFPLLVRN & WTVDXFKLKVR \\
VTWXXFKKLVS & VTVFFDPKLWRN \\
VTVDXFPKLVS & VTWXFFLPLV \\
VTVDXFPKLW & VSDXFPLLVRN \\
& \\
\hline &
\end{tabular}

Appendix A (continued)

\begin{tabular}{ll}
\hline Grammatical & Non-grammatical \\
\hline VSXFDKLPVRM & MXXFPKLWRN \\
WTDXDPKPVRN & VSXFDPPLWR \\
WSXFFKLLWRM & WSDXFKLLVRM \\
WSFFFLLLWRN & NDXFKLLWR \\
VSXXFKKLWR & WSDFDPKLWR \\
WTWXFFKLLVRM & VTWFDFLLPWS \\
VTWDXDPKPWR & MFDFPKLWR \\
VTDFFPLLWRN & NDXFKLPVRM \\
VTWXDFKPLVRN & WTVDXFKLPWR \\
WTWFFFLLLW & WTXFFLLPVR \\
WTWDFFPLLVS & WSFXFPKLWRN \\
VTXDFKPLWRN & VTXFFLLPRN \\
WSDXFPKLVRN & NXFDPKLWRN \\
VTWFFDLLPVRN & VTWDXFKKPV \\
VTWFXFLKLVRM & NXFFLPLWRN \\
NFDFLPLWR & WTWDXFKKKVRM \\
WTWXFDKLPVR & VSXDFPLPVRN \\
WTXFFKLLWRM & VTVDDFLPLWR \\
VTWDFFPLLW & VTXFFLKLVRN \\
\hline
\end{tabular}

\section{References}

Bahlmann, J., Schubotz, R. I., \& Friederici, A. D. (2008). Hierarchical artificial grammar processing engages Broca's area. NeuroImage, 42, 525-534.

Brooks, L. R., \& Vokey, J. R. (1991). Abstract analogies and abstracted grammars: Comments on Reber (1989) and Mathews et al. (1989). Journal of Experimental Psychology: General, 120, 316-323.

Caramazza, A., \& Zurif, E. (1976). Dissociations of algorithmic and heuristic processes in sentence comprehension: Evidence from aphasia. Brain and Language, 3, 572-582.

Christiansen, M. H., \& Chater, N. (2008). Language as shaped by the brain. Behavioral and Brain Sciences, 31, 489-509.

Christiansen, M. H., Louise Kelly, M., Shillcock, R. C., \& Greenfield, K. (2010). Impaired artificial grammar learning in agrammatism. Cognition, 116, 382-393.

Courtin, C., Jobard, G., Vigneau, M., Beaucousin, V., Razafimandimby, A., Herve, P. Y., et al. (2011). A common neural system is activated in hearing non-signers to process French Sign language and spoken French. Brain Research Bulletin, 84(1), 75-87.

Cristià, A., Seidl, A. \& Gerken, L. (2011). Learning classes of sounds in infancy. University of Pennsylvania Working Papers in Linguistics, 17, Article 9. Available at: <http://repository.upenn.edu/pwpl/vol17/iss11/19>.

de Vries, M. H., Barth, A. R. C., Knecht, S., Zwitserlood, P., \& Floeel, A. (2010) Electrical stimulation of Broca's area enhances implicit learning of an artificial grammar. Journal of Cognitive Neuroscience, 22, 2427-2436.

de Vries, M. H., Christiansen, M. H., \& Petersson, K. M. (2011). Learning recursion: Multiple nested and crossed dependencies. Biolinguistics, 5, 10-35.

de Vries, M. H., Monaghan, P., Knecht, S., \& Zwitserlood, P. (2008). Syntactic structure and artificial grammar learning: The learnability of embedded hierarchical structures. Cognition, 107, 763-774.

Dronkers, N. F. (2000). The gratuitous relationship between Broca's aphasia and Broca's area. Behavioral and Brain Sciences, 23, 30-31.

Dronkers, N. F., Wilkins, D. P., Van Valin, R. D. J., Redfern, B. B., \& Jaeger, J. J. (1994). A reconsideration of the brain areas involved in the disruption of morphosyntactic comprehension. Brain and Language, 47, 461-463.

Evans, J. L., Saffran, J. R., \& Robe-Torres, K. (2009). Statistical learning in children with specific language impairment. Journal of Speech, Language, Hearing Research, 52, 321-335.

Field, A. (2009). Discovering statistics using SPSS (3rd ed.). Sage Publications Ltd..

Fitch, W. T., \& Hauser, M. D. (2004). Computational constraints on syntactic processing in a nonhuman primate. Science, 303, 377-380.

Floeel, A., de Vries, M. H., Scholz, J., Breitenstein, C., \& Johansen-Berg, H. (2009). White matter integrity in the vicinity of Broca's area predicts grammar learning success. Neurolmage, 47, 1974-1981.

Folia, V., Forkstam, C., Ingvar, M., Hagoort, P., \& Petersson, K. M. (2011). Implicit artificial syntax processing: Genes, preference, and bounded recursion. Biolinguistics, 5, 105-132.

Folia, V., \& Petersson, K. M. (2014). Implicit structured sequence learning: An fMRI study of the structural mere-exposure effect. Frontiers in Psychology, 5, 41.

Folia, V., Uddén, J., De Vries, M. H., Forkstam, C., \& Petersson, K. M. (2011). Artificial language learning in adults and children. Language Learning, 60, 188-220.

Folia, V., Udden, J., Forkstam, C., Ingvar, M., Hagoort, P., \& Petersson, K. M. (2008). Implicit learning and dyslexia. Annals of the New York Academy of Sciences, 1145, 132-150. 
Forkstam, C., Elwér, Å., Ingvar, M., \& Petersson, K. M. (2008). Instruction effects in implicit artificial grammar learning: A preference for grammaticality. Brain Research, 1221, 80-92.

Forkstam, C., Hagoort, P., Fernandez, G., Ingvar, M., \& Petersson, K. M. (2006). Neural correlates of artificial syntactic structure classification. Neurolmage, 32, 956-967.

Forkstam, C., \& Petersson, K. M. (2005). Towards an explicit account of implicit learning. Current Opinion in Neurology, 18, 435-441.

Friederici, A. D., Bahlmann, J., Heim, S., Schubotz, R. I., \& Anwander, A. (2006). The brain differentiates human and non-human grammars: Functional localization and structural connectivity. Proceedings of the National Academy of Sciences of the United States of America, 103, 2458-2463.

Gerken, L. (2006). Decisions, decisions: Infant language learning when multiple generalizations are possible. Cognition, 98, B67-B74.

Gervain, J., Nespor, M., Mazuka, R., Horie, R., \& Mehler, J. (2008). Bootstrapping word order in prelexical infants: A Japanese-Italian cross-linguistic study. Cognitive Psychology, 57, 56-74.

Gomez, R. L., \& Gerken, L. (2000). Infant artificial language learning and language acquisition. Trends in Cognitive Sciences, 4, 178-186.

Gómez, R. L., \& Maye, J. (2005). The developmental trajectory of nonadjacent dependency learning. Infancy, 7, 183-206.

Grodzinsky, Y. (2000). The neurology of syntax: Language use without Broca's area. Behavioral and Brain Sciences, 23, 1-21.

Hagoort, P. (2005). On Broca, brain, and binding: A new framework. Trends in Cognitive Sciences, 9, 416-423.

Hoen, M., Golembiowski, M., Guyot, E., Deprez, V., Caplan, D., \& Dominey, P. F. (2003). Training with cognitive sequences improves syntactic comprehension in agrammatic aphasics. NeuroReport, 14, 495-499.

Janata, P., \& Grafton, S. T. (2003). Swinging in the brain: Shared neural substrates for behaviors related to sequencing and music. Nature Neuroscience, 6, 682-687.

Kinder, A., \& Lotz, A. (2009). Connectionist models of artificial grammar learning: What type of knowledge is acquired? Psychological Research PRPF, 73, 659-673.

Knowlton, B. J., \& Squire, L. R. (1996). Artificial grammar learning depends on implicit acquisition of both abstract and exemplar-specific information. Journal of Experimental Psychology: Learning, Memory, and Cognition, 22, 169-181.

Koechlin, E., \& Jubault, T. (2006). Broca's area and the hierarchical organization of human behavior. Neuron, 50, 963-974.

Lenhard, W., \& Lenhard, A. (Producer) (2016, 28/06). Calculations of effect sizes. Retrieved from <http://www.psychometrica.de/effect_size.html>.

Lieberman, M. D., Chang, G. Y., Chiao, J., Bookheimer, S. Y., \& Knowlton, B. J. (2004). An event-related fMRI study of artificial grammar learning in a balanced chunk strength design. Journal of Cognitive Neuroscience, 16, 427-438.

Linebarger, M. C., Schwartz, M., \& Saffran, E. (1983). Sensitivity to grammatical structure in so-called agrammatic aphasics. Cognition, 13, 361-393.

Maess, B., Koelsch, S., Gunter, T. C., \& Friederici, A. D. (2001). Musical syntax is processed in Broca's area: An MEG study. Nature Neuroscience, 4, 540-545.

Makuuchi, M., Bahlmann, J., Anwander, A., \& Friederici, A. D. (2009). Segregating the core computational faculty of human language from working memory. Proceedings of the National Academy of Sciences of the United States of America, $106,8362-8367$.

Martin Wetzel, W. F., Blossom-Stach, C., \& Feher, E. (1989). Syntactic loss versus processing deficit: An assessment of two theories of agrammatism and syntactic comprehension deficits. Cognition, 32, 157-191.

Meulemans, T., \& Van der Linden, M. (1997). Associative chunk strength in artificial grammar learning. Journal of Experimental Psychology: Learning, Memory, and Cognition, 23, 1007-1028.

Misyak, J. B., Christiansen, M. H., \& Tomblin, J. B. (2009). Statistical learning of nonadjacencies predicts on-line processing of long-distance dependencies in natural language. Proceedings of the Cognitive Science Society, 2009, 177-182.

Newport, E. L., Hauser, M. D., Spaepen, G., \& Aslin, R. N. (2004). Learning at a distance II. Statistical learning of non-adjacent dependencies in a non-human primate. Cognitive Psychology, 49, 85-117.

Nieuwenhuis, I. L., Folia, V., Forkstam, C., Jensen, O., \& Petersson, K. M. (2013). Sleep promotes the extraction of grammatical rules. PLoS ONE, 8(6), e65046.

Opitz, B., \& Friederici, A. D. (2007). Neural basis of processing sequential and hierarchical syntactic structures. Human Brain Mapping, 28, 585-592.
Opitz, B., \& Hofmann, J. (2015). Concurrence of rule-and similarity-based mechanisms in artificial grammar learning. Cognitive psychology, 77, 77-99.

Patel, A. D. (2003). Language, music, syntax and the brain. Nature Neuroscience, 6, 674-681.

Patel, A. D., Iversen, J., Wassenaar, M., \& Hagoort, P. (2008). Musical syntactic processing in agrammatic Broca's aphasia. Aphasiology, 22, 776-789.

Petersson, K. M., Folia, V., \& Hagoort, P. (2010). What artificial grammar learning reveals about the neurobiology of syntax. Brain and Language, 120, 83-95.

Petersson, K. M., Forkstam, C., \& Ingvar, M. (2004). Artificial syntactic violations activate Broca's region. Cognitive Science, 28, 383-407.

Petersson, K. M., \& Hagoort, P. (2012). The neurobiology of syntax: Beyond string sets. Philosophical transactions of the Royal Society of London. Series B, Biological sciences, 367, 1971-1983.

Pothos, E. M., \& Bailey, T. M. (2000). The role of similarity in artificial grammar learning. Journal of Experimental Psychology: Learning, Memory, and Cognition, 26, 847.

Pothos, E. M., \& Wood, R. L. (2009). Separate influences in learning: Evidence from artificial grammar learning with traumatic brain injury patients. Brain Research, 1275, 67-72.

Ravignani, A., Sonnweber, R. S., Stobbe, N., \& Fitch, W. T. (2013). Action at a distance: Dependency sensitivity in a New World primate. Biology Letters, 9(6).

Reber, A. S. (1967). Implicit learning of artificial grammars. Journal of Verbal Learning E Verbal Behavior, 6, 855-863.

Reber, A. S., Walkenfeld, F. F., \& Hernstadt, R. (1991). Implicit and explicit learning: Individual differences and IQ. Journal of Experimental Psychology: Learning, Memory, and Cognition, 17(5), 888-896.

Richardson, J., Harris, L., Plante, E., \& Gerken, L. (2006). Subcategory learning in normal and language learning-disabled adults: How much information do they need? Journal of Speech, Language, and Hearing Research, 49, 1257-1266.

Santelmann, L. M., \& Jusczyk, P. W. (1998). Sensitivity to discontinuous dependencies in language learners: Evidence for limitations in processing space. Cognition, 69, 105-134.

Segaert, K., Menenti, L., Weber, K., Petersson, K. M., \& Hagoort, P. (2011). Shared syntax in language production and language comprehension: An fMRI study. Cerebral Cortex. In press.

Sonnweber, R., Ravignani, A., \& Fitch, W. T. (2015). Non-adjacent visual dependency learning in chimpanzees. Animal Cognition.

Stadler, M. A., \& Frensch, P. A. E. (1998). Handbook of implicit learning. Thousand Oaks: CA: Sage Publications.

Straube, B., He, Y. F., Steines, M., Gebhardt, H., Kircher, T., Sammer, G., et al. (2013). Supramodal neural processing of abstract information conveyed by speech and gesture. Frontiers in Behavioral Neuroscience, 7.

Tessier, A.-M. (2007). Biases and stages in phonological acquisition Unpublished PhD dissertation. Amherst, MA: UMass Amherst.

Tillmann, B., Koelsch, S., Escoffier, N., Bigand, E., Lalitte, P., Friederici, A. D., et al (2006). Cognitive priming in sung and instrumental music: Activation of inferior frontal cortex. NeuroImage, 31, 1771-1782.

Uddén, J., Araujo, S., Forkstam, C., Ingvar, M., Hagoort, P., \& Petersson, K. M. (2009). A matter of time: Implicit acquisition of recursive sequence structures. Proceedings of the Cognitive Science Society, 2009, 2444-2449.

Uddén, J., \& Bahlmann, J. (2012). A rostro-caudal gradient of structured sequence processing in the left inferior frontal gyrus. Philosophical Transactions of the Royal Society of London. Series B, Biological Sciences, 367, 2023-2032.

Udden, J., Folia, V., Forkstam, C., Ingvar, M., Fernandez, G., Overeem, S., et al. (2008). The inferior frontal cortex in artificial syntax processing: An rTMS study. Brain Research, 1224, 69-78.

Uddén, J., Ingvar, M., Hagoort, P., \& Petersson, K. M. (2012). Implicit acquisition of grammars with crossed and nested non-adjacent dependencies: Investigating the push-down stack model. Cognitive Science, 36, 1078-1101.

Vosse, T., \& Kempen, G. (2000). Syntactic structure assembly in human parsing: A computational model based on competitive inhibition and a lexicalist grammar. Cognition, 75, 105-143.

Whitmarsh, S., Udden, J., Barendregt, H., \& Petersson, K. M. (2013). Mindfulness reduces habitual responding based on implicit knowledge: Evidence from artificial grammar learning. Consciousness and Cognition, 22(3), 833-845.

Willems, R. M., \& Hagoort, P. (2007). Neural evidence for the interplay between language, gesture, and action: A review. Brain and Language, 101, 278-289. 